\title{
Breast involvement in granulomatosis with polyangiitis: a case report
}

\author{
Bhavya Basetti (i), Gokulakrishnan Periakaruppan, Arunan Murali, Bhawna Dev, \\ Prabhu Radhan Radhakrishnan and P. M. Venkata Sai
}

\begin{abstract}
Background: Granulomatosis with polyangiitis (GPA) previously known as Wegener's granulomatosis is a rare complex immune-mediated disease and a multisystem disorder with varying presentations where many of the imaging features of GPA overlap with those of other diseases, and hence its diagnosis can be challenging.

Case presentation: Here we report a case of a 48-year-old female who presented with complaints of hemoptysis, difficulty in speech, bilateral progressive hearing loss and, a palpable lump in the right breast. The case was initially misdiagnosed as breast carcinoma but biopsy proved to be GPA. Here we present the multisystem manifestations of GPA involving the head and neck, breast, lungs, spleen with involvement of the breast being very rare. The breast lesion showed improvement with steroids on follow-up.

Conclusions: GPA is a multisystem disease and though breast lesions in GPA are rare, a thorough knowledge about this uncommon manifestation is required and clinical, radiological, and histopathological correlation is of utmost importance in the evaluation of a breast lesion in cases of multisystem involvement to avoid unnecessary surgeries. The differential diagnosis of breast tumors should include such rare conditions that can sometimes mimic breast cancer. Early diagnosis and initiation of the immunosuppressive treatment help in a better prognosis of the disease.
\end{abstract}

Keywords: GPA-Granulomatosis with polyangiitis, C-ANCA-Antineutrophil cytoplasmic antibody, Cavitating lung lesions

\section{Background}

Granulomatosis with polyangiitis (GPA) previously known as Wegener's granulomatosis is a rare complex immune-mediated disease, predominantly involving the respiratory and genitourinary tract. It is a multisystem disorder with varying presentations where many of the imaging features of GPA overlap with those of other diseases, and hence its diagnosis can be challenging. The diagnosis of GPA is often delayed because of vague symptoms like upper respiratory infection, sinusitis, and otitis media which may be neglected [1]. The radiologist needs to know the imaging features for early diagnosis, resulting in earlier treatment and avoid needless surgeries.

\footnotetext{
*Correspondence: bhavya.basetti@gmail.com

Department of Radiology, Sri Ramachandra Institute of Higher Education and Research, Chennai, Tamil Nadu, India
}

Here we report a case of a 48-year-old female with multisystem manifestations of GPA involving the head and neck, breast, lungs, and spleen.

\section{Case presentation}

A 48-year-old female with no known comorbidities presented to our hospital with chief complaints of hemoptysis, intermittent headache, hoarseness of voice with difficulty in speech and pain in the throat, and bilateral progressive hearing loss. She also had a painless lump in the right breast, bilateral lower limb swelling with purple-colored spots over the legs. However, there was no history of fever, cough, breathlessness, or chest pain.

On physical examination, her vitals were found to be within normal limits. There was bilateral pitting pedal edema with palpable purpura over the bilateral lower limbs (Fig. 1). In addition to this, there was a palpable 

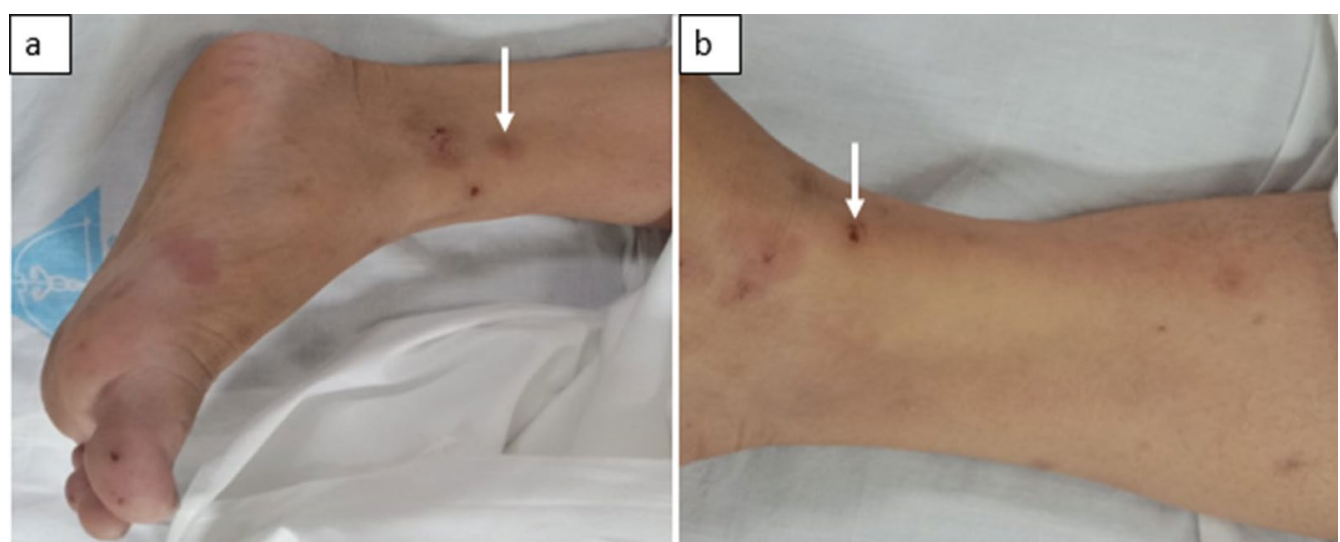

Fig. 1 Clinical examination showed palpable purpuric spots (arrows in $\mathbf{a}, \mathbf{b}$ ) on bilateral lower limbs

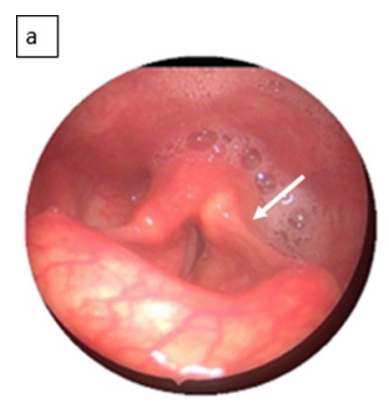

Fig. 2 Video laryngoscopy image showing left vocal cord palsy (arrow in $\mathbf{a}$ ) and pooling of saliva in the left pyriform fossa (asterisk in b)

lump in the right breast; however, the rest of her systemic examination was normal.

On routine blood investigations, the total cell count was elevated with a predominance of neutrophils (76\%). Her erythrocyte sedimentation rate $(89 \mathrm{~mm} / \mathrm{h}), \mathrm{C}$-reactive protein $(46.8 \mathrm{mg} / \mathrm{L})$ and D- Dimer values $(4.6 \mu \mathrm{g} /$ $\mathrm{ml})$ were elevated. The liver and renal function tests were found to be within normal limits. The blood and urine cultures were negative and urine analysis was positive for erythrocytes and showed hyaline casts.

Given the history of hoarseness of voice and difficulty in hearing, the patient was referred to the ENT department. A video laryngoscopy was done, which revealed left vocal cord palsy with pooling of saliva in the left pyriform fossa (Fig. 2). Bilateral otitis media was also noted at the same time.

A contrast-enhanced computed tomography (CECT) of the thorax was arranged because of elevated D-dimers and hemoptysis and it revealed few welldefined, heterogeneously enhancing lung parenchymal lesions of varying sizes and the largest of these lesions was measuring $7.0 \times 6.6 \times 6.0 \mathrm{~cm}$ with central cavitation in the right lower lobe (Fig. 3). Few enlarged lymph nodes were noted in the right upper para-tracheal, pre vascular, and sub-carinal regions. An irregular soft tissue density mass lesion was seen in the right breast measuring $2.2 \times 3.7 \mathrm{~cm}$. There was no infiltration into the skin and chest wall. Subsequently, a right breast ultrasound was done (Fig. 4) which revealed an irregular, ill-defined, hypoechoic mass lesion of size $2.0 \times 3.0 \mathrm{~cm}$ with posterior acoustic shadowing in the lower inner quadrant of the right breast. The lesion was highly suspicious for malignancy and classified as $U-$ BIRADS 4c for which a PET/CT scan and tissue biopsy were recommended.

The PET/CT scan (Fig. 5) revealed mild soft tissue thickening with increased metabolism in the posterolateral wall of the nasopharynx, nasal septum, and lingual tonsils and in the left carotid space near the skull base causing compression on the left recurrent laryngeal nerve and left vocal cord palsy. Additionally, multilobulated soft tissue density lesion with increased metabolic activity was seen in the right breast (SUV max-4.96) along with hypermetabolic soft tissue density lesions in bilateral lungs, largest lesion (SUV max6.95) showing central cavitation in the right lower lobe. Furthermore, there was splenomegaly with diffuse ill-defined hypermetabolic nodular lesions (Fig. 6). Overall, the PET/CT imaging findings were suggestive of inflammatory/ granulomatous etiology. Follow-up image-guided biopsies were done from the lung and breast lesions.

CT-guided biopsy of the right lung lesion and tru-cut biopsy of the right breast lesion was done (Fig. 7) The histopathological examinations of the right lung and breast mass lesions confirmed necrotizing granulomatous inflammation (Fig. 8) and no evidence of malignancy. Furthermore, immunohistochemistry revealed 

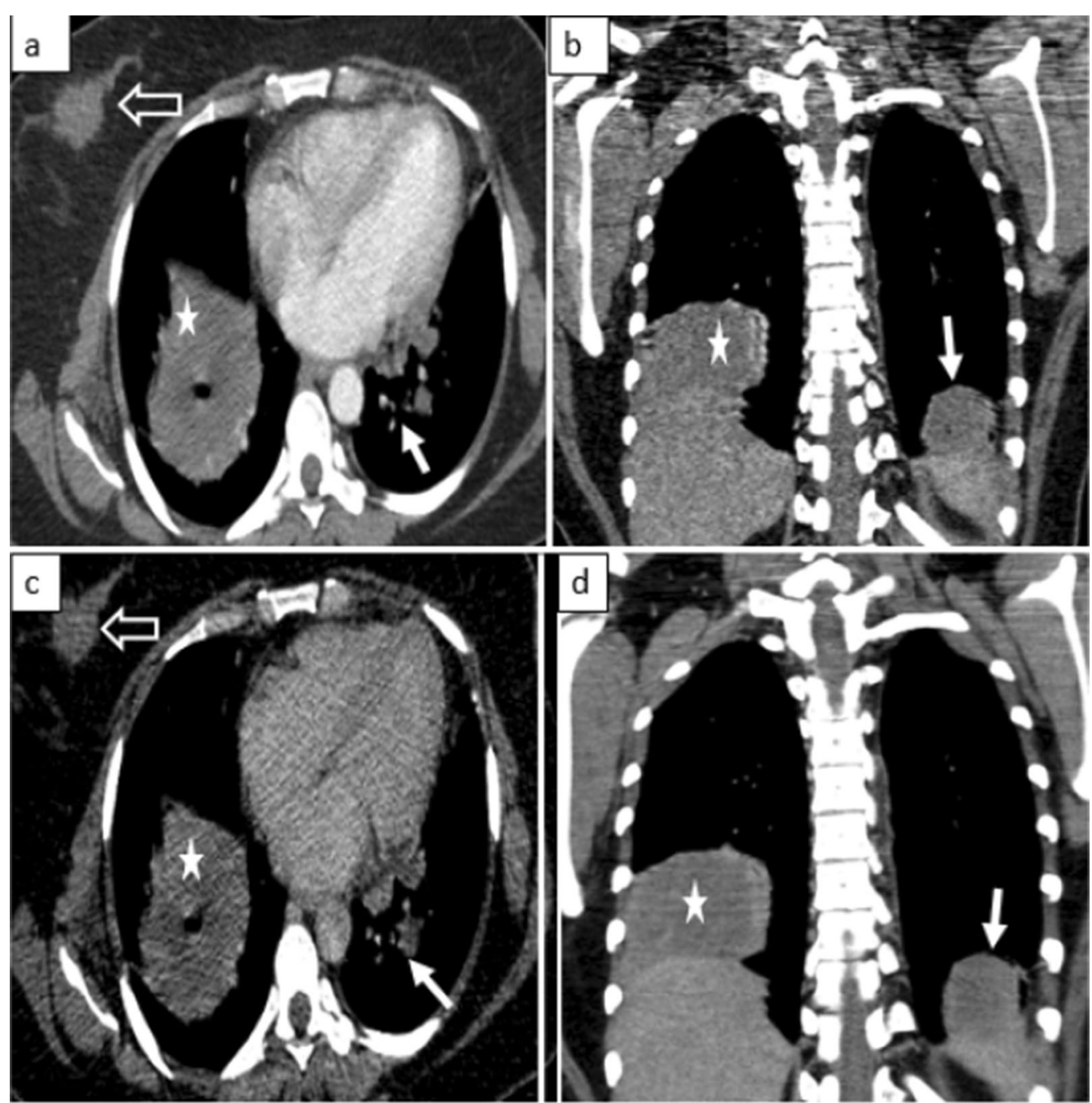

Fig. 3 (a-d) Axial and coronal sections of contrast-enhanced CT thorax of 48-year female in arterial (a, b) and venous phase (c, d) show well-defined, heterogeneously enhancing lung parenchymal lesions of varying sizes (thin arrow, star) and the largest of these lesions with central cavitation seen in the right lower lobe (star). A heterogeneously enhancing lesion noted in the right breast (open arrow in $\mathbf{a}, \mathbf{c}$ )

elevated PR3-C ANCA (> $200 \mathrm{RU} / \mathrm{ml})$. ANCA by immunofluorescence showed positivity for C-ANCA while P-ANCA and antinuclear antibodies were negative.

Hence, the patient was treated with 3 pulses of Methyl Prednisolone followed by 1 dose of IV cyclophosphamide. A follow-up breast ultrasound done after two months showed resolving mastitis (Fig. 9). However no follow-up CT was done to check for the resolution of the other lesions.

\section{Discussion}

Patients may develop GPA at any age, with the mean age being 50 years, with a slight male predominance and majority of the cases ( 90\%) in whites [1]. However, the incidence of GPA has been increasing annually, which may be a result of increased environmental factors including cadmium, silica, mercury, sand dust, and volatile hydrocarbons [2].

GPA is characterized by chronic necrotizing granulomatous inflammation of small and medium-sized vessels of the upper airway (characterized by sinusitis, otitis, nasal mucosa ulcers, bone deformities, and subglottic stenosis), lower respiratory tract (cough, chest pain, hemoptysis), and glomerulonephritis (hematuria/ proteinuria) forming the triad [2]. It has multisystem involvement with the majority involving the lung and kidney. Other organs involved are salivary glands, heart, central nervous system, gastrointestinal tract, spleen. Although only $40 \%$ of cases present with renal involvement, with the progression of the disease, almost $80-90 \%$ of cases can 

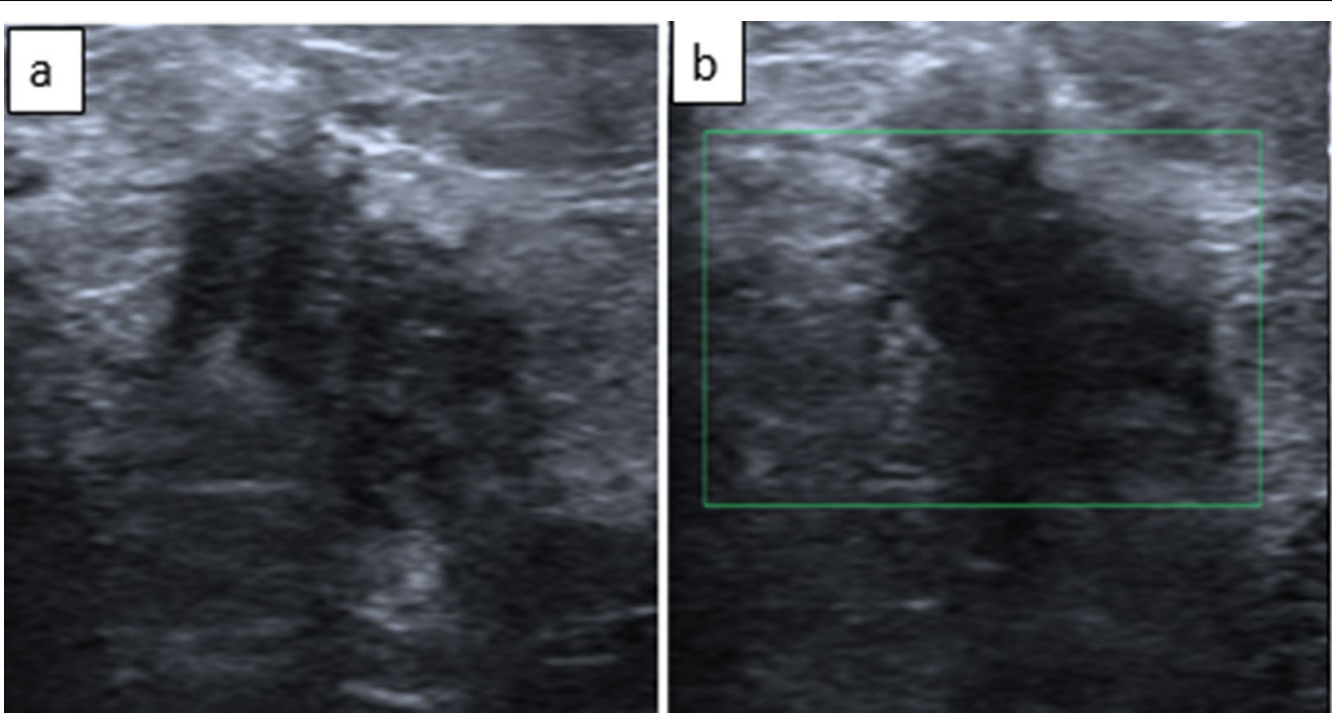

Fig. 4 Right breast ultrasound shows an irregular, ill-defined hypoechoic lesion with non-parallel orientation showing posterior acoustic shadowing in the lower inner quadrant of the right breast (a). No evidence of internal vascularity (b). Features were highly suspicious of malignancy and the lesion was characterized as U- BIRADS 4C
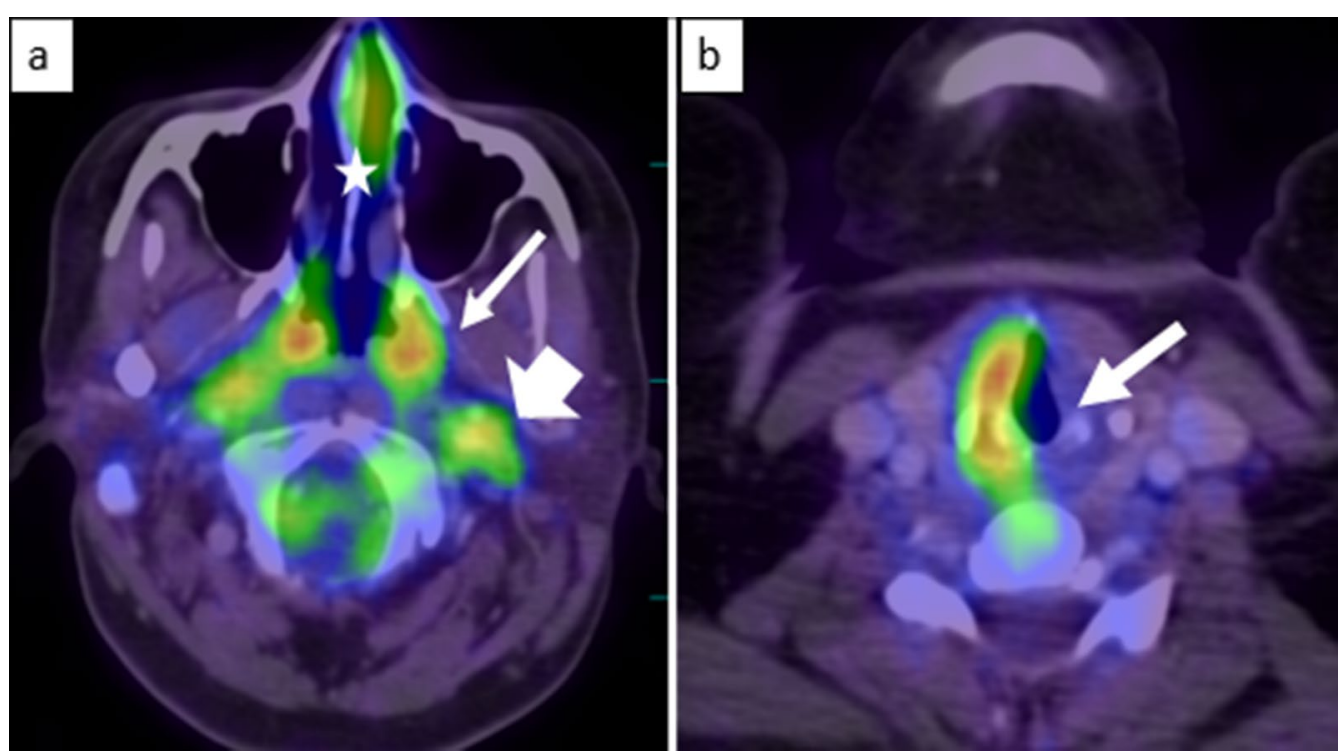

Fig. 5 Axial PET/CT image a shows soft tissue thickening with increased metabolism in the posterolateral wall of the nasopharynx bilaterally (thin arrow), nasal septum (star) and in the left carotid space near the skull base (thick arrow). Axial PET/CT image $\mathbf{b}$ shows adducted left vocal cord (arrow)

be involved [3]. However, the clinical presentation of the disease depends on the organ system involved.

Pathologically, GPA is characterized by necrotizing non-caseating granulomatous inflammation of small vessel walls, resulting in areas of necrosis surrounded by hemorrhage, small microabscesses, and granulomata within the lungs [4]. Serological examination shows normocytic anemia, leucocytosis, elevated erythrocyte sedimentation rate (ESR), antineutrophil cytoplasmic antibody (specifically PR3-ANCA), and positive rheumatoid factor). C-ANCA is positive in $90 \%$ of the systemic forms and $60 \%$ of the limited forms of the disease. P-ANCA is reactive in a minority of cases. The PR3ANCA enzyme-linked immunosorbent assay is a recently introduced test with a sensitivity of $96 \%$ and a specificity of $98.5 \%$ [5].

Diagnostic criteria were established by the American College of Rheumatology in 1990 to confirm GPA 


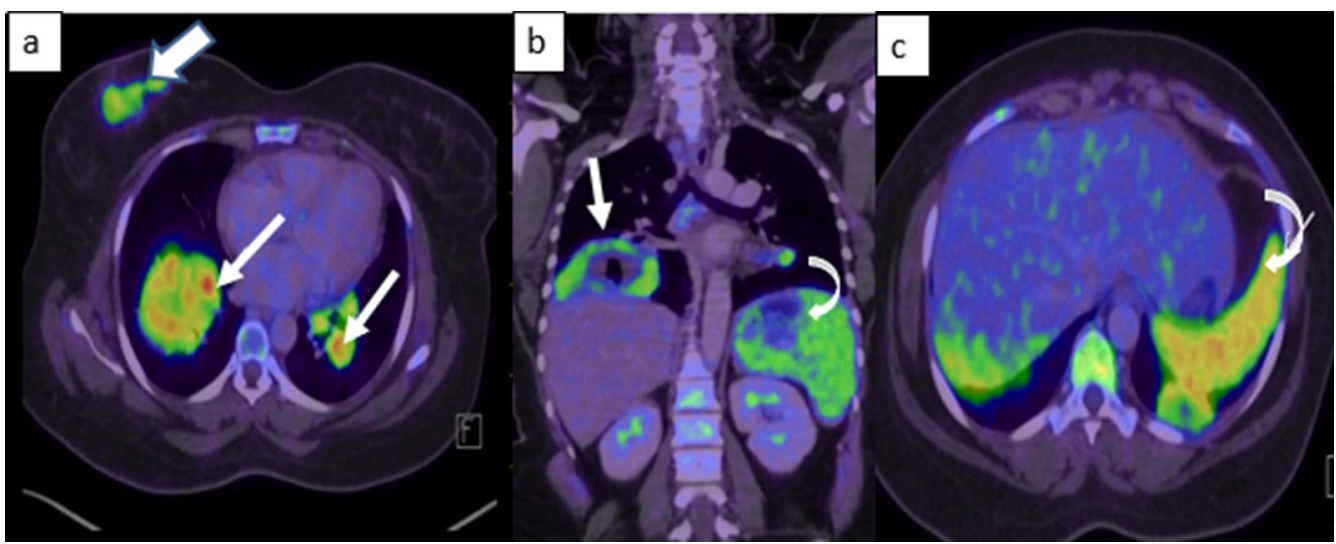

Fig. 6 (a-c) Axial and coronal sections of PET/CT a-c show multilobulated soft tissue density lesion with increased metabolic activity in the right breast (thick arrow) with hypermetabolic soft tissue density lesions in bilateral lungs, largest lesion showing central cavitation in the right lower lobe (thin arrow). There was splenomegaly with diffuse ill-defined hypermetabolic nodular lesions (curved arrow)
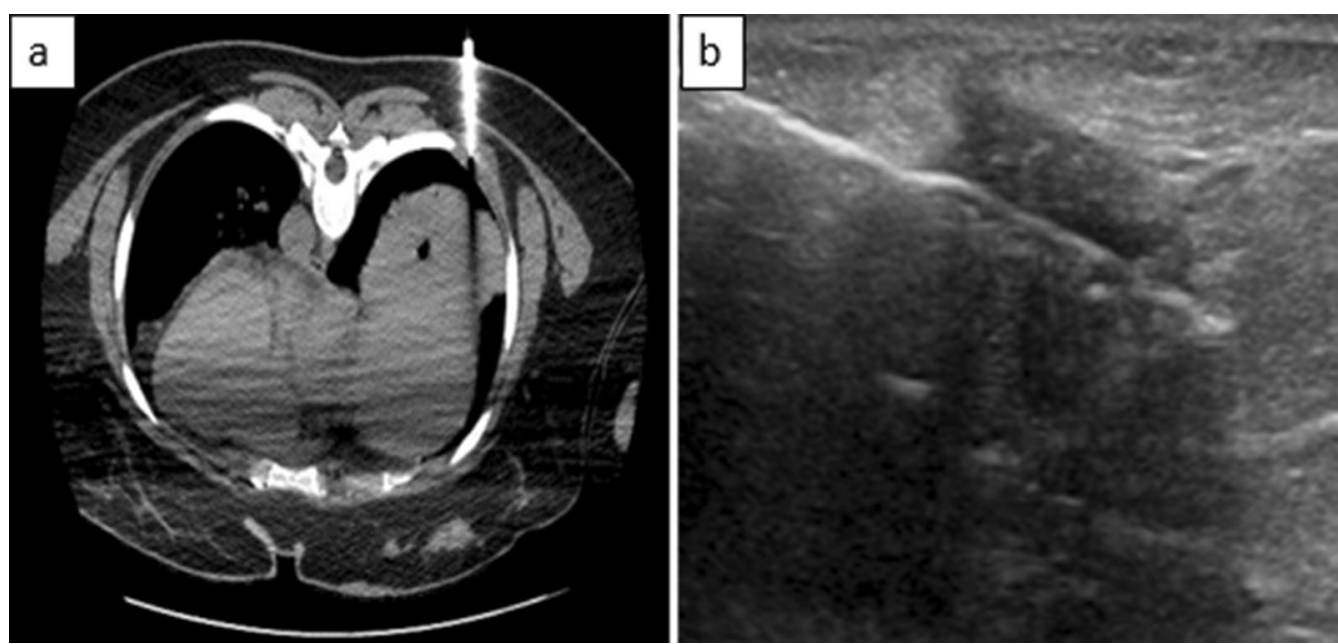

Fig. 7 CT guided biopsy of right lung lesion (a) and tru-cut biopsy of the right breast lesion (b) was done

(Table 1). The presence of two or more of the four criteria is associated with a sensitivity of $88.2 \%$ and a specificity of $92 \%$ for Wegener granulomatosis [6]. Multisystem imaging features in GPA include:

- Pulmonary manifestations: It is seen in about $95 \%$ of cases and presents with multiple lung nodules and masses ranging from few millimeters to 10 centimetres in size (usually $2-4 \mathrm{~cm}$ ) and may show random distribution when multiple. In nodules greater than $2 \mathrm{~cm}$, central cavitation is more common. Cavity walls may be smooth and thin or irregular and thick and can be mistaken for septic emboli, metastasis, and lung abscess [7]. Lung consolidation and

Table 1 Diagnostic criteria for confirmation of GPA according to the American College of Rheumatology

\begin{tabular}{ll}
\hline Criteria & Characteristics \\
\hline Nasal or oral inflammation & Painful or painless oral ulcers, purulent or bloody nasal discharge \\
Abnormal chest radiograph & Nodules, fixed infiltrates, cavities \\
Urinary sediment & Microhematuria (>5 RBCs per high power field), red cell cast \\
Granulomatous inflammation at biopsy & Involvement of the wall of an artery/ arteriole, involvement of \\
& the perivascular / extravascular spaces \\
\hline
\end{tabular}




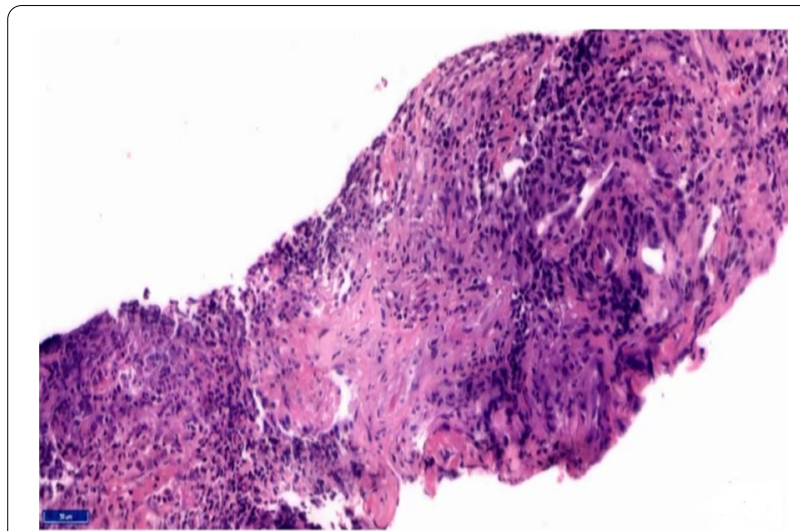

Fig. 8 Histopathology revealed liquefactive necrosis in lungs with profuse eosinophils, multinucleated giant cells, as part of poorly formed granulomas, surrounded by palisading histiocytes and giant cells with central necrosis along with destructive leukocytic angiitis of arteries and veins suggestive of granulomatosis polyangiitis with necrosis nia reaction in the periphery of focal hemorrhage [7].

- Airway involvement: The subglottic portion of the trachea is most often affected and can be segmental, unifocal, or multifocal involving a $2-4 \mathrm{~cm}$ span. Wall thickening is usually circumferential and can be smooth or nodular and the posterior membrane of the trachea is involved [2].

- Nasal cavity and paranasal sinuses: Nonspecific sinonasal mucosal thickening with nasal septal thinning and granulomatous nodules are routinely seen. Bony and cartilaginous erosions, mainly in the nasal septum are the most common feature in GPA, giving a saddle nose deformity [4].

- Renal manifestations: Wegener's granulomatosis in the kidney is characterized by focal and segmental glomerulonephritis, sometimes accompanied by a necrotizing granulomatous vasculitis resembling
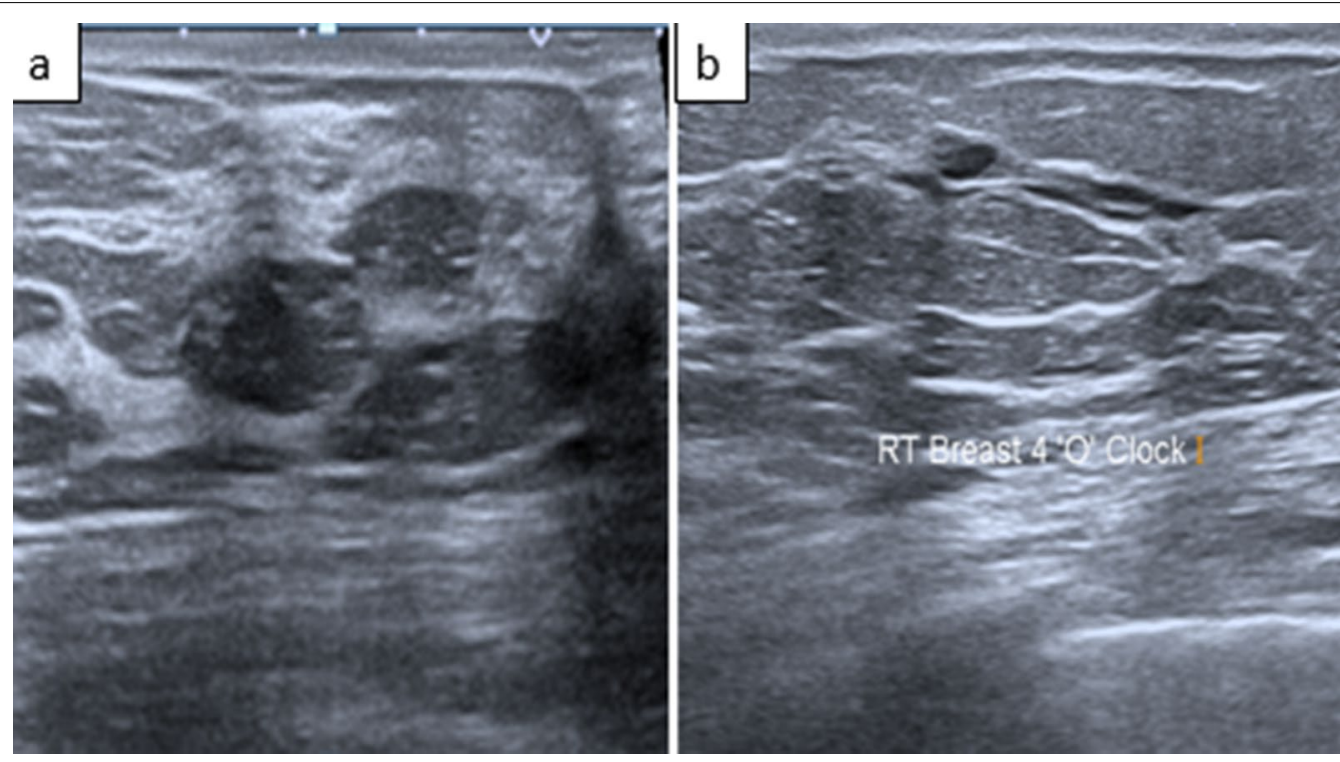

Fig. 9 Follow-up breast ultrasound $\mathbf{a}, \mathbf{b}$ after 2 months of immunosuppressant therapy show features of resolving mastitis

ground-glass opacity often occur in approximately $30 \%$ of patients with active Wegener's granulomatosis and are usually the result of hemorrhage. Hemorrhage may occur around nodules and manifests ground-glass opacity surrounding the consolidated nodule, referred to as the "halo sign". A "reverse halo sign" may also occur in Wegener's granulomatosis presumably reflecting an organizing pneumo- renal tumors [4].

- Middle ear and skull base: Middle ear involvement is due to direct extension of adjacent sinonasal disease and obstruction of the Eustachian tube and ultimately resulting in serous otitis media, the most common otologic finding. CT may show opacification of the bilateral middle ears and mastoid air 
cells with thickening of the bony septa [8]. Skull base involvement in GPA commonly results from direct extension of the neighboring granulomatous sinonasal or orbital lesion and causes thickening and enhancement of the adjacent cranial nerves, mainly the olfactory nerve and optic nerve [8].

- Spleen: Though splenic involvement is rare in GPA segmental splenic infarction may occur due to diffuse arteritis resulting in occlusion of distal parenchymal splenic arteries and show peripheral wedge-shaped areas of low attenuation on CT [4].

- Breast: Breast involvement by GPA is very unusual. Elsner et al. first reported this in 1969 [9]. There are only 36 cases reported so far in the English literature [10]. Allendes et al. [5] described differentiation of GPA from other causes of granulomatous mastitis in their extensive review on breast lesions in GPA in 2009. GPA of the breast can have a varied presentation and can occur both in males and females in the form of palpable single or multiple nodules, with or without fixation of the overlying skin. Other reports mention nodular lesions and multifocal skin ulcerations, tender breast mass with fever, retraction of the nipple, thickening of the skin, and clear discharge from the nipple [11]. In rare situations, a breast lump is the only presenting symptom. Imaging features include an ill-defined irregular mass or focal asymmetric density on a mammogram and as an irregular hypoechoic nodular mass or mass with parenchymal mixed echogenicity, consistent with mastitis or abscess on ultrasound examination [12]. In such cases, clinicians may consider a malignancy in the differential diagnosis and may lead to an unnecessary breast biopsy and surgery. Several granulomatous diseases can be infrequent causes of granulomatous inflammation of the breast like tuberculosis, sarcoidosis, syphilis, fungal infections, and granulomatous mastitis. The vasculitis variants that may involve the breast are polyarteritis nodosa, giant-cell arteritis, scleroderma, and Behçet's disease [13].

In our case, upper and lower respiratory tract symptoms were seen along with the cavitating lung mass. The breast lesion was initially misdiagnosed as a metastatic lesion. However, the biopsy revealed necrotizing granulomatous mastitis.

\section{Management}

Combination of cyclophosphamide with corticosteroids is the treatment of choice in severe life-threatening disease whereas a combination of methotrexate with corticosteroids is used in patients without the life-threatening disease [14].

\section{Conclusions}

GPA is a multisystem disease and though breast lesions in GPA are rare, a thorough knowledge about this uncommon manifestation is required and clinical, radiological, and histopathological correlation is of utmost importance in the evaluation of a breast lesion in cases of multisystem involvement and avoids unnecessary surgeries. The differential diagnosis of breast tumors should include such rare conditions that can sometimes mimic breast cancer. Early diagnosis and initiation of the immunosuppressive treatment help in the better prognosis of the disease.

\section{Abbreviations}

GPA: Granulomatosis with polyangiitis; C-ANCA: Antineutrophil cytoplasmic antibody.

\section{Acknowledgements}

The author would like to thank her great and beloved teachers, her closest friends and her mentors for their aids in making her complete this article.

\section{Authors' contributions}

BB did the major write up of this case report. The case in this case report was diagnosed and followed up by GP and AM. The work was carried under the guidance GP and provided us the insight and knowledge to diagnose indeterminate lesions with imaging alone. BD performed the breast ultrasound and biopsy and gave further inputs about the case. PR followed up the case with respective biochemical investigations and HPE results. All the authors read the rough draft and provided valuable suggestions for the final draft. VS reviewed this article for corrections and final draft. All authors read and approved the final manuscript.

\section{Funding}

This article did not receive any specific grant from funding agencies in the public, commercial, or not-for-profit sectors.

\section{Availability of data and materials}

The data are taken solely from our institution.

\section{Declarations}

Ethics approval and consent to participate

Written informed consent to publish this information was obtained from study participant.

\section{Consent for publication}

Patient included in this case report gave written informed consent to publish the data contained within this study.

\section{Competing interests}

The authors declare that they have no competing interests.

Received: 18 May 2021 Accepted: 26 July 2021

Published online: 03 August 2021

\section{References}

1. Olivencia-Simmons I (2007) Wegener's granulomatosis: symptoms, diagnosis, and treatment. J Am Acad Nurse Pract 19(6):315-320. https://doi. org/10.1111/j.1745-7599.2007.00231.x 
2. Martinez F, Chung JH, Digumarthy SR, Kanne JP, Abbott GF, Shepard JA, Mark EJ, Sharma A (2012) Common and uncommon manifestations of Wegener granulomatosis at chest CT: radiologic-pathologic correlation. Radiographics 32(1):51-69. https://doi.org/10.1148/rg.321115060

3. Chen Y, Ding Y, Zhengzhao L, Zhang H, Liu H, Hu W (2016) Long-term outcomes in antineutrophil cytoplasmic autoantibody-positive eosinophilic granulomatosis with polyangiitis patients with renal involvement: a retrospective study of 14 Chinese patients. BMC Nephrol. https://doi.org/ 10.1186/s12882-016-0319-2

4. Allen SD, Harvey CJ (2007) Imaging of Wegener's granulomatosis. Br J Radiol 80(957):757-765. https://doi.org/10.1259/bjr/34705892

5. Allende DS, Booth CN (2009) Wegener's granulomatosis of the breast: a rare entity with daily clinical relevance. Ann Diagn Pathol 13(5):351-357. https://doi.org/10.1016/j.anndiagpath.2009.04.005

6. Fries JF, Hunder GG, Bloch DA, Michel BA, Arend WP, Calabrese LH, Fauci AS, Leavitt RY, Lie JT, Lightfoot RW Jr (1990) The American College of Rheumatology 1990 criteria for the classification of vasculitis Summary. Arthritis Rheum 33(8):1135-1136. https://doi.org/10.1002/art.1780330812

7. Ananthakrishnan L, Sharma N, Kanne JP (2009) Wegener's granulomatosis in the chest: high-resolution CT findings. AJR Am J Roentgenol 192(3):676-682. https://doi.org/10.2214/AJR.08.1837

8. Pakalniskis MG, Berg AD, Policeni BA, Gentry LR, Sato Y, Moritani T, Smoker WR (2015) The many faces of granulomatosis with polyangiitis: a review of the head and neck imaging manifestations. AJR Am J Roentgenol 205(6):W619-W629. https://doi.org/10.2214/AJR.14.13864

9. Elsner B, Harper FB (1969) Disseminated Wegener's granulomatosis with breast involvement. Report of a case. Arch Pathol 87(5):544-547
10. Gadeyne L, Henckaerts L, Goffin KE, Gheysens O, Lerut E, Roskams T, Blockmans D, Floris G (2019) Granulomatosis with polyangiitis with breast involvement mimicking metastatic cancer: case report and literature review. Eur J Rheumatol 7(1):41-43. https://doi.org/10.5152/eurjrheum. 2019.19065

11. Neralić-Meniga I, Ivanovi-Herceg Z, Mazuranić I, Puljić I, Zekan M, Gorecan M, Kos M (2006) Wegener's granulomatosis of the breast. Wien Klin Wochenschr 118(3-4):120-123. https://doi.org/10.1007/ s00508-006-0536-y

12. Georgescu R, Podeanu MD, Colcer I, Grigorescu G, Coroș MF, Moldovan C, Ilyes A, Bârsan I, Moncea D, Stolnicu S (2015) Wegener's granulomatosis of the breast with peculiar radiological aspect mimicking breast carcinoma. Breast J 21(5):550-552. https://doi.org/10.1111/tbj.12458

13. Goulabchand R, Hafidi A, Van de Perre P, Millet I, Maria A, Morel J, Quellec AL, Perrochia H, Guilpain P (2020) Mastitis in autoimmune diseases: review of the literature, diagnostic pathway, and pathophysiological key players. J Clin Med 9(4):958. https://doi.org/10.3390/jcm9040958

14. White ES, Lynch JP (2006) Pharmacological therapy for Wegener's granulomatosis. Drugs 66(9):1209-1228. https://doi.org/10.2165/00003 495-200666090-00004

\section{Publisher's Note}

Springer Nature remains neutral with regard to jurisdictional claims in published maps and institutional affiliations.

\section{Submit your manuscript to a SpringerOpen ${ }^{\circ}$ journal and benefit from:}

- Convenient online submission

$\checkmark$ Rigorous peer review

- Open access: articles freely available online

- High visibility within the field

- Retaining the copyright to your article

Submit your next manuscript at $\boldsymbol{\nabla}$ springeropen.com 